\title{
Dal dialogo all'assunzione della propria condizione
}

\author{
Enrico Vincenti*
}

\section{Introduzione}

Occuparsi del disagio e della sofferenza nel cosiddetto 'ambito psicotico', pone una serie di questioni: il primo riguarda la fragilità e delicatezza della persona sofferente, il modo in cui vengono espresse, il loro intersecarsi con la fragilità e le difficoltà di coloro a cui essa è affettivamente legata. Il secondo riguarda i professionisti della 'salute mentale', i luoghi di 'cura', fino a toccare la dimensione contestuale, culturale e organizzativa dei Servizi.

Seikkula, in questo articolo, descrive l'iter che ha portato alla definizione di un modello di intervento in psichiatria, di organizzazione dei Servizi e di formazione del personale tendente a dar voce alle persone; rivolto all'ascolto di tutti coloro che sono toccati dalla sofferenza. Una visione, come sostiene l'autore, che non guarda alla psicopatologia e alla diagnosi, ma al paziente e alla persona. Ci presenta una dimensione etica, di ascolto e rispetto della persona, andando oltre l'affermazione di una competenza tecnico-operativa. Di conseguenza, l'attenzione non è rivolta alla diagnosi per procedere alla definizione della terapia e dell'intervento, ma la diagnosi emergerebbe dall'ascolto della voce del paziente e dei familiari. La naturale ricaduta di un approccio di questo tipo mi sembra porti verso una 'umanizzazione della psichiatria e dei servizi ad essa dedicati'.

Proviamo a seguire il percorso descritto da Seikkula e, mi riservo in conclusione, alcune considerazioni sulla proposta.

\section{Periodo storico}

Apprendo che i primi passi del modello risalgono agli anni ' 60 , in coincidenza con l'esigenza di trasferire dall'ospedale psichiatrico di Tornio,

\footnotetext{
*Psicologo e Psicoterapeuta, Supervisore SIPRe - IFPS. Docente presso le sedi di Milano e Parma della Scuola di Psicoterapia ad indirizzo Psicoanalisi della Relazione.

E-mail: evince57@gmail.com
} 
alcune decine di pazienti lungodegenti considerati 'incurabili'. La prima questione che ci si pose, rispetto alla 'incurabilità' di alcune patologie psichiatriche, riguardava lo studio della correlazione tra esiti dell'intervento e 'filosofia della cura'. L'idea di fondo portava a ritenere che l'occuparsi dei sintomi della sofferenza psichiatrica avrebbe aperto la porta al non ascolto e alla etichettatura del paziente e, quindi di fatto, avrebbe partecipato involontariamente alla sua cronicizzazione.

Invece, si cercò di verificare come un atteggiamento differente del curante, rivolto all'ascolto della persona, avrebbe potuto contribuire alla costruzione di una prassi e di un 'modello di trattamento più ottimistico', uscendo dalla visione riduttiva di una relazione lineare tra cura e sintomo. $\mathrm{Si}$ arrivò così ad occuparsi non solo della sofferenza espressa dal paziente e, non ci si limitò al trattamento farmacologico e al rapporto terapeutico individuale, si allargò il campo includendo nel percorso di cura anche una dimensione familiare e quindi contestuale. Mi sembra che questo progetto psichiatrico, sebbene non espresso dall'autore, si inserisse all'interno di un movimento internazionale di critica alla concezione organica della sofferenza umana; un tentativo di ascolto della persona, della sua voce e del suo dolore esistenziale; provando così a dare dignità alla sofferenza accogliendone le differenti e singolari espressioni.

Per far questo era necessario uscire dalla vecchia logica della chiusura della sofferenza in luoghi ristretti e angusti: chiudendo in quelle mura tutto ciò che risultava estraneo, incomprensibile e non conforme alla cultura imperante. Non conosco il contesto finlandese e non so se, anche in quel territorio, esistevano gli ospedali psichiatrici e se i cosiddetti 'lungodegenti incurabili' erano rappresentati da persone 'pericolose per sé e per gli altri e/o che danno offesa all'ordine pubblico' tanto da essere internate con decreto del giudice o della polizia. In Italia era così e da lì è nata l'esigenza di messa in crisi del sistema psichiatrico e della ideologia sottostante, con la nascita di movimenti di 'liberazione della psichiatria' e di democratizzazione della sua prassi. Movimento che in Italia ha portato nel 1978 alla emanazione della 'legge 180' detta 'legge Basaglia', in onore del maggior rappresentante di quel movimento.

'Nascevano allora i Servizi psichiatrici di Diagnosi e Cura (SPDC) allocati all'interno degli Ospedali Generali, Centri di Salute Mentale, Centri di Crisi e strutture intermedie di cura collocate sul territorio comunale (Case-famiglia, Centri Diurni, Appartamenti Protetti). L'ideale basagliano, che professa la 'liberazione'del malato psichiatrico, è naturalmente connesso alla 'liberazione' della psichiatria, delle sue pratiche e valori, cosi come della società; il motto 'Liberare Liberandosi' conteneva l'idea di una netta connessione tra le diverse forze sociali, ed esprimeva la necessità di non segregare il disagio dal sociale, poiché sarebbe stata una soluzione semplicistica e violenta. Era necessario portare la sofferenza nel sociale, ricongiungerlo alla famiglia, al lavoro, alla società' (Vincenti \& Irtelli, 2018). 
Espressione di una psichiatria non 'oppressiva e potenzialmente iatrogena', non costituita da mura e contenimento, ma una psichiatria aperta al territorio e al sociale, quindi una psichiatria che si differenziava dalla concezione medica e si avvicinava alle scienze umane e sociali. Una psichiatria che si occupava dell'essere umano sofferente nel suo contesto, ritenendo l'umano non solo organico ma anche sociale e relazionale.

Psichiatri, psicoanalisti, operatori che guardavano con occhi differenti alla manifestazione umana della sofferenza psichiatrica; un occhio non rivolto alla sintomatologia e alla diagnosi, non intento a debellare il sintomo, ma impegnato, insieme al paziente, a darne un senso come manifestazione dell'essere umano degno di essere rispettato nella sua essenza.

Come scrive Borgna:

\begin{abstract}
'La psichiatria è una disciplina ambigua e dilemmatica che, quando sia aperta agli orizzonti interdisciplinari della ricerca, non si occupa solo delle malattie (dei disturbi psichici), ma si confronta con i fenomeni del mondo della vita, astraendo dal loro essere normali, o patologici, e in particolare con gli arcipelaghi sconfinati delle emozioni. Certo, se nel fare psichiatria non siamo capaci di immedesimarci nella vita interiore degli altri, degli altri che stanno male in particolare, e non siamo capaci di intuizioni..., non ci è possibile cogliere fino in fondo il senso del dolore e della sofferenza, della tristezza e dell'angoscia, degli smarrimenti e delle lacerazioni dell 'anima, del silenzio e dello stupore del cuore, che fanno parte della condizione umana nelle sue infinite forme di espressione'(Borgna, 2019).
\end{abstract}

\title{
La proposta
}

A partire dalla intuizione di Alanen di un trattamento 'adattato al bisogno', dove si contemplavano differenti metodi terapeutici, utilizzati di volta in volta a seconda delle esigenze specifiche della situazione, viene a organizzarsi l'approccio 'Dialogo Aperto', intendendo il 'modo in cui è organizzato il sistema psichiatrico, sia al ruolo del dialogo negli incontri con i pazienti, familiari e gli operatori'. Quindi due differenti dimensioni interconnesse: uno rivolto al paziente, l'altro al Servizio; entrambe unite dall'intento di favorire il dialogo tra le diverse componenti: nella cura tra paziente, familiari e rete; nel Servizio tra utenti, equipe e rete allargata.

Il paziente viene attivamente coinvolto assieme ai suoi familiari nella definizione delle problematiche e nella ricerca di soluzioni; esso è parte attiva nel processo di cura e, come sosteneva Alanen (riportato da Seikkula), l'attenzione viene rivolta al rafforzamento del lato adulto del paziente e alla normalizzazione della situazione, invece di concentrarsi sul comportamento regressivo. In particolare, si combina una attenzione individuale al paziente, tramite una terapia dinamica individuale e una attenzione alla famiglia, al contesto, tramite un intervento ad orientamento sistemico familiare. Seikkula 
ci tiene a sottolineare che l'attenzione del terapeuta non è rivolta alla creazione di specifiche forme di conduzione del colloquio, ma è orientato all'ascolto e alla risposta. Un ascolto non razionale ma empatico, partecipato 'in effetti, sembra che il passaggio dal discorso monologico rigido e ristretto al dialogo avvenga da sé quando le emozioni dolorose non sono trattate come pericolose, ma invece lasciare fluire liberamente nella stanza'.

Di conseguenza l'invito e la formazione degli operatori verte proprio sul lasciarsi andare ad una partecipazione attiva rispondendo, da persona a persona, a quanto l'interlocutore porta, anzi partendo proprio dalle sue parole. Un rapporto dialogico in cui si realizza, con la propria presenza incarnata, un atteggiamento di massimo ascolto dell'altro, espresso sia in forma verbale sia non verbale; quindi, ascolto e "normalizzazione 'di ciò che si dice anche in forma psicotica. Questo modo di procedere risponde al presupposto teorico, ripreso da Bakhtin, 'essere significa comunicare dialogicamente... nel dialogo una persona non solo si mostra esteriormente, ma diventa per la prima volta ciò che è... non solo per gli altri ma anche per sé stesso'.

In fondo c'è un a priori: il paziente sta male perché non riesce a dire e dare un senso a quanto è accaduto nella sua vita. Una sorta di situazione traumatica che l'intervento basato sul dialogo fa sì che il paziente, $i$ familiari e la rete, in connessione tra loro, riescano a riprendere e a dare un senso attraverso una condivisione emotiva dell'esperienza. Si sottolinea molto l'importanza della partecipazione e condivisione emotiva, attuata attraverso il congiungersi ciascuno con le proprie tematiche dolorose. Il riuscire a stare su queste tematiche e nell'incertezza avrebbe finalità terapeutiche. Sicuramente un andare oltre il modello medicalista basato sul sintomo da eliminare e sul rapporto tra un professionista esperto 'che sa' e un paziente ignaro da curare. Un modello aperto in cui anche il professionista e i componenti della rete sono invitati a mettersi in gioco in prima persona.

Sebbene condivida la necessità di uscire da un modello organicistico e unidirezionale della cura, non mi ritrovo completamente nel modello presentato da Seikkula. Non perché ritengo inutile la condivisione e la partecipazione del contesto e della rete al progetto terapeutico, ma per l'idea teorica a monte. Seikkula ci propone un modello che tiene conto sicuramente della complessità, tanto da aver concepito l'importanza del tener presente il soggetto e le sue relazioni, non fermandosi solo ad una analisi della comunicazione e delle relazioni, ma cercando di dare senso al mondo del paziente. Questa idea di fondo e la conseguente pratica, sicuramente hanno portato a dei grossi passi avanti nella concezione della psicosi come disagio e sofferenza umana, non riducibile alla sola componente organica e biologica. Un pensiero che porta ad una visione positiva sia della persona sia delle possibilità evolutive nelle situazioni di disagio.

Penso comunque sia necessario fare un passo ulteriore per valorizzare al massimo il contributo dell'autore. Prendere in considerazione il contesto, 
permette di inserire la persona sofferente all'interno del suo ambiente di vita e quindi, dal mio punto di vista, permette di cogliere nelle sue relazioni la funzionalità che esse hanno nel mantenere le soluzioni storiche che l'hanno configurato. Diversi autori, come Maturana, hanno indagato l'organizzazione e il funzionamento dell'essere vivente inserito nel proprio ambiente e Oyama ha esplicitato come i processi di auto-eco organizzazione e delle ricorsività circolari sono alla base della sua esistenza. Sander (2003) il primo psicoanalista che ha tentato di trasporre questi concetti all'essere umano, afferma che non si può pensare 'ad alcun organismo vivente... senza pensare anche ad un ambiente in cui esso debba essere in continua interazione... come un 'sistema': l'organismo e il suo ambiente'. A partire da questi autori, possiamo cercare di ipotizzare come si sia organizzato e come funziona un essere umano. Se l'essere umano si auto-eco-organizza all'interno del suo ambiente, possiamo pensare che è anche quell'ambiente, che l'ha definito e che anche lui contribuisce a definire. Da ciò ne consegue che le relazioni di ciascun soggetto sono funzionali a mantenere la propria configurazione, attraverso continui processi di circolarità ricorsiva.

A questo livello, che Minolli definisce di 'coscienza' (quindi a un livello di processamento analogo a tutti gli esseri viventi), la relazione, qualsiasi tipo di relazione, è funzionale per ogni partecipante nel mantenere la propria configurazione e nell'affermare sé stesso. Nel perseguimento di questo fine vitale, spesso si verificano attribuzioni di 'delega' all'altro. Intendendo con delega tutto ciò che il soggetto utilizza quando fa fatica a stare in piedi da solo, a prendere in mano la propria vita, la propria storia e le proprie relazioni.

Seikkula ci offre una descrizione esemplare delle attribuzioni di delega reciproca quando scrive:

'Diversi membri della rete vivono in situazioni diverse, persino contraddittorie, e quindi possono avere idee molto diverse sul problema. Considerate una crisi che coinvolge una madre, un padre e un figlio, in cui il figlio, sospettato di abuso di droghe, diventa quasi psicotico. Il padre può essere preoccupato principalmente per la reputazione della famiglia tra i suoi colleghi e la madre per la salute di suo figlio, e il giovane può protestare con rabbia che non ha bisogno di alcun trattamento e che $i$ suoi genitori sono pazzi e dovrebbero cercare cure per sé stessi.'

Dove emerge con chiarezza quanta fatica fanno i tre ad occuparsi della propria esistenza, con la conseguenza che ciascuno attribuisce all'altro la responsabilità delle proprie difficoltà e del proprio malessere. Coinvolgerli insieme potrebbe essere occasione, non solo di condividere e trovare significati prima inaccessibili, ma soprattutto per aiutarli a fare i conti con i reciproci appoggi, le richieste e pretese verso l'altro, aiutandoli a riconoscere le attribuzioni di delega e a provare a stare in piedi da soli avendo fiducia in sé stessi. 
Un discorso analogo, sull'utilizzo delle deleghe, potremmo farlo per quanto riguarda l'equipe curante. Attribuire e, intestardirsi ad attribuire, le difficoltà della persona che si rivolge al Servizio ad una causa organica, cercando di eliminare il sintomo, per esempio con ansiolitici o antipsicotici, potrebbero rappresentare una difficoltà a mettersi in gioco, ad accogliere la sofferenza che il nostro interlocutore ci porta. Pretesa che viene formalizzata e istituzionalizzata all'interno della visione teorica e del metodo di trattamento, dove esiste 'l'esperto' che sa e il paziente 'ignaro' che necessita di essere curato.

Credo sia naturale operare in questo modo poiché esso risponde all'esigenza vitale del soggetto di affermare ciò che lui è per come si è andato configurando all'interno del suo specifico contesto.

Questa esigenza vale per tutti: paziente (colui che chiede o per cui si chiede l'intervento), familiari e professionisti della salute. Nel senso che tutti sono impegnati nel vitale compito di continuare la propria esistenza affermando ciò che sono, per come si sono venuti configurando in base al patrimonio genetico ricevuto dai propri genitori, alle relazioni significative e al contesto in cui si sono trovati. Occuparsi di queste relazioni, tenendo presente la funzionalità delle stesse, rispettando l'organizzazione e le soluzioni di ciascuno, in quanto funzionali per poter vivere, poiché sono le soluzioni migliori che ciascuno ha trovato per poter esistere, è la premessa per un intervento rispettoso.

Ascoltare il paziente, i familiari e i membri della rete concretizza un 'porsi' del professionista in una relazione vera e diretta con l'altro accogliendo la sua sofferenza, rinunciando alla posizione di potere di colui che sa e che indirizza. Credo che gli esiti presentati da Seikkula riguardo all'affievolirsi della sintomatologia, al minor ricorso dell'utilizzo di psicofarmaci e di interventi o alla necessità di ricovero, sia attribuibile al cambio di paradigma di intervento.

È una posizione scomoda perché ci si trova a mani nude con l'altro e con sé stessi. Così si concretizza la possibilità di stare nell'insicurezza, poiché si perdono i punti di appoggio, la cosiddetta 'delega', delle 'presunte 'conoscenze tecniche, per toccare con mano la sofferenza umana attraverso le soluzioni e le espressioni che essa ha in coloro che chiedono aiuto. L'impatto emotivo dello stare ha a che fare con questa dimensione della presenza. Per cui la formazione degli operatori è un passaggio fondamentale poiché è necessario accompagnarli ad abbandonare le presunte conoscenze tecniche, per acquisire una diversa consapevolezza di sé stessi, tanto da poter 'reggere' l'impatto della condizione umana, in ogni forma di espressione.

\section{L'équipe}

Visto la voglia e la difficoltà a confrontarsi con la sofferenza psicotica, nella seconda metà del secolo scorso, in molte contesti hanno sentito 
l'esigenza di coinvolgere attivamente i membri della équipe nel processo di cura e la necessità di una formazione continuativa degli operatori. Penso per esempio a Racamier e al lavoro pionieristico descritto nel 'lo psicoanalista senza divano: La psicoanalisi e le strutture psichiatriche'.

In fondo possiamo affermare che il lavoro rivolto alla équipe trovava fondamento nell'idea che le dinamiche tra gli operatori fossero espressione/proiezione del mondo interno del paziente. Per cui lo sforzo era rivolto all'analisi delle dinamiche interpersonali dei membri della équipe al fine di superare le scissioni ed avere una visione integrata del paziente. In questo modo l'équipe funzionava e veniva concepita come un 'organo' di digestione /comprensione della sofferenza per poterla poi restituire al paziente.

Seikkula propone un passo ulteriore, non è l'equipe che predigerisce quanto il paziente non riesce a digerisce per poi restituirlo al paziente, ma è l'insieme, paziente compreso, che si sforza di trovare un senso attraverso uno continuo scambio partecipato, in modo che possano nascere nuove parole e un nuovo discorso sulla sofferenza.

Ho seguito l'autore inserendo la sua proposta all'interno di una evoluzione culturale generale del mondo occidentale. Ora vorrei fare un passo ulteriore, perché se la proposta porta con sé una visione positiva del paziente, del suo coinvolgimento nella ricerca di una diversa concezione del suo malessere, il posizionare la possibilità evolutiva nel 'dialogo rispondente' lascia entrare dalla finestra ciò che si è fatto uscire dalla porta: una visione limitata dell'essere umano, condannandolo ad una dipendenza eterna dall'altro. Il dialogo rispondente potrebbe correre il rischio di attribuire la possibilità evolutiva al dialogo e alla risposta dell'altro, sottovalutando così la possibilità/capacità dell'essere umano, attraverso la coscienza della coscienza, di occuparsi della propria sofferenza, di accoglierla nella propria vita e di cercare un altro modo per andare avanti. Capacità che dobbiamo pensare essere possibile a ciascun essere umano, anche al cosiddetto paziente psicotico.

\section{La politica dei servizi}

Non mi sono soffermato sulle indicazioni tecniche e operative proposte, perché sono conseguenti alla visione teorica esposta: risposta entro le $24 \mathrm{~h}$, presa in carico, continuità e garanzia di prosieguo da parte della stessa équipe, ecc. sono espressione della necessità di occuparsi della persona sofferente.

Così come, al contrario, la non continuità della presa in carico, il passaggio da un professionista all'altro, il non contare sulla équipe o sulla continuità nel rapporto di cura, sono diretta conseguenza della visione teorica dell'essere umano, considerato come afferma Stolorow una 'mente isolata' e la sua sofferenza dovuta ad una alterazione biochimica. 
La psichiatria, come dice Borgna, è considerata una cenerentola all'interno della medicina, sopportata ma mai del tutto accettata. Anche la spesa sanitaria ad essa attribuita così come l'attenzione alla organizzazione dei Servizi lascia spesso molto a desiderare. Purtroppo, negli ultimi anni in Italia, si è visto una precarizzazione delle équipe di cura, un cambio continuo di psichiatri e operatori che difficilmente si concilia con la presa in carico della sofferenza e delle persone.

Per fortuna non tutti i servizi sono organizzati in questo modo, ci sono esempi eccellenti di utilizzo delle scarse risorse economiche in funzione della valorizzazione delle risorse umane, 'strumento' principe nella presa in carico della sofferenza psichiatrica.

Mi fa piacere sapere che in Finlandia e in alcuni paesi, la proposta di Seikkula sia attecchita, perché rappresenta uno sforzo per andare verso una positiva visione dell'uomo e del rispetto del suo modo di essere. Soprattutto di quel mondo ritenuto incomprensibile come la sofferenza 'psicotica'.

\section{BIBLIOGRAFIA}

Basaglia, F. (2000). Conferenze brasiliane. Milano: Raffaello Cortina.

Basaglia, F. (a cura) di (1968). L'istituzione negata. Torino: Einaudi.

Borgna, E. (2019). La follia che è in noi (ebook - Vele vol.149). Torino: Einaudi.

Ferro, A., Jervis, G. (1999). La bottega della psichiatria. Torino: Bollati Boringhieri.

Maturana, H. R., Varela, F. J. (1985). Autopoiesi e cognizione. La realizzazione del vivente. Venezia: Marsili.

Maturana, H. R., Varela, F.J. (1987). L'albero della conoscenza. Milano: Garzanti.

Minolli, M. (2004). Per un Io-Soggetto fatto di legami. Ricerca Psicoanalitica, XV(3), 317 329.

Minolli, M. (2015). Essere e divenire. Milano: Franco Angeli.

Oyama, S. (2004). L'occhio dell'evoluzione, tr. it. Roma: Fioriti.

Pichon-Rivière, E. (1985). Il processo gruppale, dalla psicoananlisi alla psicologia sociale, tr. it. Loreto: Lauretana.

Racamier, P. C. (1982). Lo psicoanalista senza divano. Milano: Cortina Editore.

Racamier, P. C. (1998). Una comunità di cura terapeutica, tr. it. In Ferruta, A., Foresti, G., Pedriali, E., Vigorelli M. (a cura di), La comunità terapeutica. Tra mito e realtà. Milano: Cortina Editore.

Sander, L. (2005). Pensare differentemente. Per una concettualizzazione dei processi di base dei sistemi viventi. Trad. it. Ricerca Psicoanalitica, XVI(3), 267-300.

Sander, L. (2007). Sistemi viventi. L'emergenza della persona attraverso l'evoluzione della consapevolezza, tr. it. Milano: Cortina Editore.

Stolorow, R. D., Brandchaft, B., Atwood, G. E., Fosshage, J. (1999). Psicopatologia intersoggettiva. Urbino: Quattro Venti Edizioni.

Sullivan, H. S. (1977). Teoria interpersonale della psichiatria, tr. it. Milano: Feltrinelli. 
Vincenti, E. (2006). Alcune risposte della Psicoanalisi Relazionale alle gravi configurazioni psicopatologiche. XIV International Forum of Psycoanalysis, 23-27 maggio, Roma.

Vincenti, E. (2016). Dentro e fuori la stanza d'analisi: l'intervento in una struttura residenziale psichiatrica. In Corbelli, L., Fontana, M. (a cura di), Psicoanalisi e schizofrenia. Milano: FrancoAngeli.

Vincenti, E., Irtelli, F. (2018). A quarant'anni dalla Legge 180. Ricerca Psicoanalitica, $X X I X(3), 37-51$.

Conflitto di interessi: l'autore dichiara che non vi sono potenziali conflitti di interessi.

Approvazione etica e consenso a partecipare: non necessario.

Ricevuto per la pubblicazione: 9 novembre 2021 .

Accettato per la pubblicazione: 9 novembre 2021.

${ }^{\circ}$ Copyright: the Author(s), 2021

Licensee PAGEPress, Italy

Ricerca Psicoanalitica 2021; XXXII:603

doi:10.4081/rp.2021.603

This article is distributed under the terms of the Creative Commons Attribution Noncommercial License (by-nc 4.0) which permits any noncommercial use, distribution, and reproduction in any medium, provided the original author(s) and source are credited. 
